Original paper

\title{
Clinical, serological, histopathological and treatment profile of autoimmune hepatitis in the elderly
}

\author{
Nikhil Sonthalia, Shubham Jain, Ravi Thanage, Parmeshwar Junare, Sanjay Chandnani, Vinay Pawar, Qais Contractor,
} Pravin Rathi

Topiwala National Medical College, Mumbai, India

\begin{abstract}
Aim of the study: Autoimmune hepatitis (AlH), despite being uncommon, is on the rise in the elderly population. However, no study from India has described the natural history and treatment outcome of AlH in the elderly. The aim was to study the characteristics of AlH in the elderly population and compare them with the younger population.

Material and methods: Patients with a diagnosis of AIH based on the revised International Autoimmune Hepatitis Group (IAIHG) criteria were recruited from January 2011 to June 2018. Patients were defined as elderly when $\geqslant 60$ years and young when $<60$ years of age. Clinical, serological, histological characteristics and treatment outcome with follow-up until 12 months were analyzed and compared between the two groups.

Results: Out of 155 patients, 33 (21.29\%) were elderly. Acute-on-chronic liver failure (ACLF) as the presentation was more common in elderly as compared to young AlH patients (39.4\% vs. $13.9 \%, p=0.0024)$. Serum alanine aminotransferases and serum creatinine levels were significantly higher in elderly patients as compared to the younger group $(p<0.05)$. On histology cirrhosis was significantly more common in the elderly group $(75.7 \%$ vs. $56.6 \%, p=0.045$ ). Response to treatment at the end of 12 months was similar in both groups. Due to co-morbidities immunosuppressant could not be started in $18.2 \%$ of elderly and $6.5 \%$ of younger patients $(p=0.065)$.

Conclusions: AlH is an important differential diagnosis among the elderly population presenting with ACLF and cirrhosis. When given appropriate immunosuppressants they have a similar outcome as compared to the youngest population.
\end{abstract}

Key words: autoimmune hepatitis (AIH), treatment outcomes in autoimmune hepatitis, acute-on-chronic liver failure.

\section{Address for correspondence}

Dr. Shubham Jain, Topiwala National Medical College, Mumbai central, 400008, Mumbai, India, e-mail: dr.shubhamjazz@gmail.com

\section{Introduction}

Autoimmune hepatitis (AIH) has been traditionally a disease of young women and is uncommon in the elderly population $[1,2]$. Recent epidemiological studies have shown an increase in prevalence of AIH among the elderly $[3,4]$. It is a chronic disease of the hepatic parenchyma which is characterized by circulating autoantibodies, increase in serum gamma globulins and morphological changes of interface hepatitis. The pathogenesis of AIH is still unclear with an immunological basis being the most commonly proposed mechanism. HLA DR3 and DR4 allotypes are independent risk fac- tors for varied presentation of AIH [5]. DR4 phenotype predisposes to AIH in the elderly population with a different course from that in the younger population [6]. Several studies have shown an incidence pattern of AIH with two age peaks, one in the second decade and one between the fourth and sixth decade $[7,8]$. There are only 10 studies with a small sample size reported in the literature describing AIH in the elderly, with varying results [9]. However, none are reported from India, where the overall incidence of AIH is on the rise, as reported by our group previously [10].

There has been no consensus regarding natural history and treatment outcomes in the elderly population 
with AIH because of such varied presentations reported in the limited number of studies from the West. Though there have been descriptive studies about AIH in the Indian population, there has been none specifically describing the clinical characteristics in the elderly Indian population [11]. The aim of our study was to understand the role of increasing age in presentation, natural history and response to treatment in elderly Indian patients with $\mathrm{AIH}$ and compare them with the younger population.

\section{Material and methods}

\section{Patient selection}

This was a hospital-based descriptive study where a prospectively maintained database of patients attending a tertiary care referral center in Western India from January 2011 to June 2018 was analyzed. Out of 155 consecutive patients who were diagnosed as having AIH, 33 patients who were $\geq 60$ years of age were defined as elderly AIH patients and were finally included in the analysis. Their demographic, clinical, serological, histological characteristics and treatment outcome with follow-up until 12 months were analyzed and compared with younger AIH patients.

Autoimmune hepatitis was diagnosed in those with a pretreatment revised International Autoimmune Hepatitis Group (IAIHG) score of $>15$ (definite) or post-treatment score $>17$ (definite). However, in a few patients in whom liver biopsy could not be done, treatment was given to patients having IAIHG scores of 1015 and these were labeled as probable $\mathrm{AIH}$ cases after ruling out other causes. To diagnose AIH detailed clinical evaluation followed by serological screening and histologic assessment was undertaken to exclude other causes of liver disease. These included exclusion of viral hepatitis (A, B, C, and E, Herpes simplex, cytomegalovirus), alcoholic liver disease, nonalcoholic fatty liver disease, celiac disease, and Wilson disease. Serological markers that were used to exclude other causes of liver diseases included immunoglobulin A (IgA) anti-tissue transglutaminase antibody, anti-HAV (hepatitis A virus) antibody, anti-HEV (hepatitis E virus) antibody, HBsAg antigen, anti-HBc total antibody, HBV DNA (quantitative), anti-HCV (hepatitis C virus) antibody, and HCV RNA (quantitative). Careful drug history was taken to exclude drug-induced hepatotoxicity. Ultrasonography was done in all the cases. Magnetic resonance cholangiopancreatography was performed in selected cases with cholestasis. An upper gastrointestinal endoscopy was performed to look for varices. Patients with insufficient follow-up data of less than 12 months were excluded from the analysis. Conventional autoantibodies which included anti-nuclear antibodies (ANA) and/or anti-smooth muscle antibody (ASMA), or anti-liver-kidney-microsomal antibody 1 (anti-LKM-1) and serum immunoglobulin G ( $\operatorname{IgG})$ were measured in all the patients. Apart from these, unconventional antibodies including anti-soluble liver antigen (SLA) and perinuclear anti-neutrophilic cytoplasmic antibody (p-ANCA) were tested in selected cases where conventional autoantibodies were negative. Only classic AIH was included in the analysis. Medical records of patients who met the inclusion criteria were reviewed to record demographic features, clinical features, severity, serum aminotransferase levels, serum total bilirubin, and prothrombin time with international normalized ratio (INR), serum autoantibody levels, serum immunoglobulin G levels, histologic features, and treatment regimens at presentation and at 12 months of follow-up.

Among AIH patients, in order to define the severity the following definitions were used: 1) Acute severe AIH patients were those who had an acute first presentation of disease with an INR $>1.5 ; 2$ ) ALF (acute liver failure) patients were those who had hepatic encephalopathy and coagulopathy (INR > 1.5) within 26 weeks of the onset of illness, but without previously recognized liver disease; 3) ACLF (acute on chronic liver failure) patients were those who had acute hepatic insult manifesting as coagulopathy (INR $>1.5$ or prothrombin activity $<40 \%$ ) and jaundice (serum bilirubin $>5 \mathrm{mg} / \mathrm{dl}$ ), followed by ascites and/or encephalopathy within 4 weeks in previously undiagnosed or diagnosed (including those who were nonresponders to immunosuppressants or who had recurrent disease) chronic AIH.

Informed consent was taken from the patients and the study was approved by the institutional ethics committee.

\section{Treatment protocol}

Wherever possible, all the patients received either (1) steroid + azathioprine or (2) only steroids. Reasons for giving only steroids included those positive for TPMT mutation, pregnancy, azathioprine intolerance, and fulminant hepatic failure. Drug therapy was started after excluding infection including tuberculosis. Wherever appropriate blood culture, urine culture, ascitic fluid culture, Mantoux test and chest x-ray were done. When using both the drugs, prednisolone was started at $30 \mathrm{mg}$ once daily, gradually tapering to $10 \mathrm{mg}$ once daily at the end of 4 weeks (maintenance dose), together with azathioprine at $50 \mathrm{mg}$ once daily. When only one drug was used, prednisolone was given 
at $60 \mathrm{mg}$ once daily, gradually tapering to $20 \mathrm{mg}$ daily (maintenance dose). Among elderly AIH patients supportive care was given in 6 patients who had contraindications (including severe sepsis, psychosis, grade 4 hepatic encephalopathy) or who were intolerant to immunosuppressive therapy.

\section{Treatment outcomes}

Treatment outcomes were defined as complete response, partial response (incomplete response), treatment failure, or death. Complete responders were defined as (1) those with complete resolution of clinical symptoms including jaundice and ascites, and (2) those in whom there was normalization of AST/ALT, total bilirubin, and serum IgG within 1 year of starting treatment [12]. Although ideally we must wait for 3 years before labeling patients as incomplete responders, for our study patients who had partial improvement in clinical and laboratory parameters but not complete normalization within 12 months of starting treatment were labeled as incomplete responders. Treatment failures were defined as those with failure to improve or who had worsening of any clinical and laboratory parameters within 2 weeks of starting the treatment. Those who failed to improve were treated with prednisolone again with the dosage increased to $30 \mathrm{mg}$ daily and the azathioprine dosage increased to $150 \mathrm{mg}$ daily at a fixed dose for 1 month. In those in whom azathioprine was not given, prednisolone $60 \mathrm{mg}$ daily was given. Thereafter, the patients were assessed and the doses were reduced by $10 \mathrm{mg}$ of prednisolone and $50 \mathrm{mg}$ of azathioprine after each month of laboratory and clinical improvement until the maintenance dose was reached. Among treatment failures, those who had worsening as evidenced by liver failure with worsening of MELD score within 7 days or increasing bilirubin within 2 weeks of treatment were listed for liver transplant (LT).

\section{Immunoserological analysis}

Anti-nuclear antibodies, ASMA, and anti-mitochondrial antibodies (AMA) were measured through indirect immunofluorescence (Euroimmun, Germany). Patients with a dilution of $\geq 1$ : 40 were considered as positive. Anti-LKM-1, anti-SLA, and p-ANCA were assessed by immunoblot assays (EUROASSAY; Euroimmun). An immunoglobulin assay was performed using the method of immunologic turbidimetry (Roche Diagnostics, Germany). Hepatitis B markers and antibodies to hepatitis $\mathrm{C}$ were assessed using second-generation ELISAs.

\section{Histologic evaluation}

Liver biopsies were available in all the 33 elderly AIH patients and in 113/122 younger AIH patients. Among elderly AIH patients most of them (19/33) underwent a standard percutaneous liver biopsy. Transjugular liver biopsy (10/33) and plugged-percutaneous liver biopsy $(4 / 33)$ techniques were used in patients with ascites and coagulopathy where the standard method could not be used. All the samples were obtained before initiation of AIH therapy. Sections were stained with hematoxylin eosin and Masson trichrome for all samples. Two experienced histopathologists, who were blinded to clinical and serological data, reviewed the histopathologic slides. For each patient between 1 and 5 slides with an average of 3 slides were available. The features that were specifically looked for included the presence of piecemeal necrosis, bridging necrosis, centrilobular necrosis, plasma cell infiltration, portal inflammatory activity, lobular inflammatory activity, Rosette formation, bile ductular injury, cholestasis, granulomas, and fibrosis. Interface hepatitis was graded as: none, minimal or mild, moderate, to severe interface hepatitis, and the fibrosis stage as: graded from no fibrosis, to minimal or mild, moderate, or cirrhosis.

\section{Statistical analysis}

All the continuous data were expressed as mean with SD or percentages with confidence intervals. For each categorical variable's association with the type of disease the $\chi^{2}$ test was used. The Mann-Whitney $U$ test and Student's $t$ test were used to compare nonparametric and parametric continuous variables, respectively. A $p$-value $<0.05$ was considered significant and all reported $p$ values were 2 -tailed. All the variables that had significant associations $(p<0.05)$ were included for multivariate logistic regression analysis with an entry probability of 0.05 . All statistical analyses were performed using SPSS statistics software.

\section{Results}

Out of 155 patients, 33 (21.29\%) were elderly. Females were predominant in the younger group as well as in the elder group ( $92.6 \%$ vs. $75.8 \%, p>0.05$ ). Fatigue, abdominal pain, fever, and arthralgia were significantly more common in younger $\mathrm{AIH}$ patients compared to elderly ones. Jaundice was more common in young $\mathrm{AIH}$ patients, whereas hematemesis and encephalopathy were more common in elderly patients, although the difference was not significant. Spontaneous bacterial peritonitis was significantly more common in the elderly. An overview of the laboratory tests at presentation is given in Table 1 . 
Table 1. Baseline demographic, clinical, and serological parameters and treatment given in ( $<60 \mathrm{vs.}>60$ years)

\begin{tabular}{|c|c|c|c|c|}
\hline \multirow[t]{2}{*}{ Parameter } & \multicolumn{2}{|c|}{ Age (years) } & \multirow[t]{2}{*}{ Total } & \multirow[t]{2}{*}{$P$-value } \\
\hline & $<60$ & $>60$ & & \\
\hline Female & $113(92.6 \%)$ & $25(75.8 \%)$ & $137(88.4 \%)$ & 0.0059 \\
\hline \multicolumn{5}{|l|}{ Clinical features } \\
\hline Jaundice & $91(74.6 \%)$ & $22(66.7 \%)$ & $113(72.9 \%)$ & 0.364 \\
\hline Hematemesis & $20(16.4 \%)$ & $9(27.3 \%)$ & $29(18.7 \%)$ & 0.155 \\
\hline Edema symptom & $66(54.1 \%)$ & $20(60.6 \%)$ & $86(55.5 \%)$ & 0.505 \\
\hline Fatigue & $86(70.5 \%)$ & $14(42.4 \%)$ & $100(64.5 \%)$ & $0.003^{*}$ \\
\hline Hepatic encephalopathy & $12(9.8 \%)$ & $5(15.2 \%)$ & $17(11.0 \%)$ & 0.386 \\
\hline Pruritus & $29(23.8 \%)$ & $4(12.1 \%)$ & $33(21.3 \%)$ & 0.147 \\
\hline Abdominal pain & $48(39.3 \%)$ & $6(18.2 \%)$ & $54(34.8 \%)$ & $0.024^{*}$ \\
\hline Fever symptom & $38(31.1 \%)$ & $4(12.1 \%)$ & $42(27.1 \%)$ & $0.029 *$ \\
\hline Arthralgia & $58(47.5 \%)$ & $7(21.2 \%)$ & $65(41.9 \%)$ & $0.007^{*}$ \\
\hline Skin rash & $18(14.8 \%)$ & $2(6.1 \%)$ & $20(12.9 \%)$ & 0.186 \\
\hline \multicolumn{5}{|l|}{ Signs } \\
\hline Hepatomegaly & $47(38.5 \%)$ & $4(12.1 \%)$ & $51(32.9 \%)$ & $0.004^{*}$ \\
\hline Splenomegaly & 78 (63.9\%) & $15(45.5 \%)$ & $93(60.0 \%)$ & 0.055 \\
\hline Ascites & $62(50.8 \%)$ & $19(57.6 \%)$ & $81(52.3 \%)$ & 0.491 \\
\hline \multicolumn{5}{|c|}{ Associated autoimmune disease } \\
\hline Diabetes mellitus & $23(18.9 \%)$ & $10(30.3 \%)$ & $33(21.3 \%)$ & 0.154 \\
\hline Thyroiditis & $37(30.3 \%)$ & $3(9.1 \%)$ & $40(25.8 \%)$ & $0.013^{*}$ \\
\hline Rheumatoid arthritis & $6(4.9 \%)$ & $2(6.1 \%)$ & $8(5.2 \%)$ & 0.792 \\
\hline Vitiligo & $3(2.5 \%)$ & $1(3.0 \%)$ & $49(2.6 \%)$ & 0.854 \\
\hline \multicolumn{5}{|l|}{ Laboratory parameters } \\
\hline Hemoglobin (gm/dl) & $9.96 \pm 2.06$ & $9.66 \pm 1.12$ & & 0.426 \\
\hline Platelets $\left(\mathrm{mm}^{3}\right)$ & $168142.62 \pm 152450.55$ & $123000 \pm 33506.529$ & & 0.094 \\
\hline Total bilirubin (mg\%) & $7.96 \pm 8.87$ & $10.45 \pm 12.96$ & & 0.203 \\
\hline AST (IU/I) & $189.22 \pm 184.91$ & $191.73 \pm 170.02$ & & 0.944 \\
\hline ALT (IU/I) & $125.60 \pm 118.505$ & $183.30 \pm 176.29$ & & $0.028^{*}$ \\
\hline $\operatorname{ALP}(I U / I)$ & $209.80 \pm 141.52$ & $206.94 \pm 161.99$ & & 0.912 \\
\hline Serum albumin (gm/dl) & $2.96 \pm 0.66$ & $2.82 \pm 0.38$ & & 0.220 \\
\hline Serum globulin (gm/dl) & $3.57 \pm 0.78$ & $3.5 \pm 0.72$ & & 0.608 \\
\hline INR & $1.35 \pm 0.44$ & $1.25 \pm 0.36$ & & 0.276 \\
\hline Creatinine (mg/dl) & $0.85 \pm 0.31$ & $1.23 \pm 0.56$ & & 0.0001 * \\
\hline MELD score at baseline & $14.78 \pm 6.14$ & $15.52 \pm 7.85$ & & 0.566 \\
\hline \multicolumn{5}{|l|}{ Serological patterns } \\
\hline ANA positive & $77(73.3 \%)$ & $28(84.48 \%)$ & $105(67.7 \%)$ & 0.195 \\
\hline ASMA positive & $31(25.4 \%)$ & $15(45.5 \%)$ & $46(29.7 \%)$ & 0.042 \\
\hline Anti LKM-1 & $5(4.1 \%)$ & $1(3 \%)$ & $6(3.9 \%)$ & 0.778 \\
\hline P-ANCA & $7(5.7 \%)$ & 0 & $7(4.5 \%)$ & 0.064 \\
\hline Anti SLA & $2(1.6 \%)$ & 0 & $2(1.3 \%)$ & 0.459 \\
\hline$\overline{A M A}$ & $10(8.2 \%)$ & $1(3 \%)$ & $11(6.5 \%)$ & 0.3052 \\
\hline \multicolumn{5}{|l|}{ Treatment given } \\
\hline Only steroid & $11(9.01 \%)$ & $4(12.12 \%)$ & & 0.838 \\
\hline Steroid plus azathioprine & $103(84.42 \%)$ & $23(69.7 \%)$ & & 0.15 \\
\hline Not given & $8(6.5 \%)$ & $6(18.18 \%)$ & & 0.857 \\
\hline
\end{tabular}

*Significant p values. ACLF indicates acute-on-chronic liver failure; AIH - autoimmune hepatitis, ALF - acute liver failure, ALT - alanine aminotransferase, AMA - antimitochondrial antibody, ANA - antinuclear antibody, anti-SLA - anti-soluble liver antigen, anti-LKM-1 - anti-liver kidney microsomal antibody type 1, ASMA - anti-smooth muscle antibody, AST - aspartate aminotransferase, IgG - immunoglobulin G, INR - international normalized ratio, MELD - Modified End-Stage Liver Disease score, p-ANCA - peripheral anti-neutrophilic cytoplasmic antibody 
Alanine aminotransferases and serum creatinine levels were significantly higher in elderly patients as compared to the younger group $(p<0.05)$, whereas INR and bilirubin values were similar in the two groups. Acute kidney injury was more common in the elderly group. Among autoimmune markers, ASMA was significantly more commonly seen in the elderly ( $45.5 \%$ vs. $25.4 \%, p<0.05)$ whereas ANA and mean serum immunoglobulin levels were present similarly in both the groups. Other associated autoimmune diseases, especially autoimmune thyroiditis, were more common in the younger population.

Acute-on-chronic liver failure as the presentation of AIH was more common in elderly as compared to young AIH patients $(39.4 \%$ vs. $13.9 \%, p=0.0024)$. The chronic presentation was more common in the younger age group $(73.8 \%$ vs. $54.5 \%, p=0.033)$. However, presentation as acute hepatitis was similar in both the groups (Fig. 1).

The frequency of key histologic features in the young and elderly group is depicted in Table 2.

Out of 155 patients, 146 underwent liver biopsy; therefore these 9 patients who were all in the younger AIH group were labeled as the probable AIH category based on their IAIHG score. Biopsies were available in all elderly patients.

On histology evidence of interface hepatitis, lymphoplasmacytic infiltrate, and Rosette formation was similar in both groups. Cirrhosis was significantly more common in the older group $(75.7 \%$ vs. $56.6 \%$, $p=0.045)$.

Due to increased prevalence of co-morbidities including severe osteoporosis appropriate immunosuppressants could not be started in $18.2 \%$ of elderly patients as compared to $6.5 \%$ of younger patients $(p=0.065)$. Steroids with azathioprine for 12 months could be given in $66.7 \%$ of elderly AIH patients as compared to $78.7 \%$ of younger AIH patients. In others only steroids were given. Among the 114 younger patients with $\mathrm{AIH}$ in whom immunosuppressants could be started, 75 (65.7\%) achieved remission,

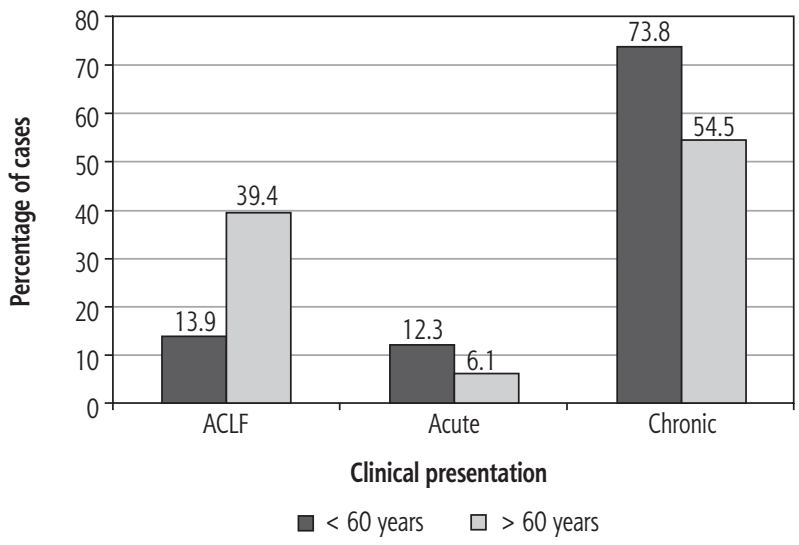

Fig. 1. Bar diagram depicting various presentations of autoimmune hepatitis (AIH) among young and older groups. This bar diagram shows that ACLF-like presentation was more common in the older AlH group (39.4\% vs. $13.9 \%$, $p=0.0024)$, whereas chronic presentation was more common in the younger group $(73.8 \%$ vs. $54.5 \%, p=0.033)$. Acute hepatitis like presentation was similar in both the groups

35 (30.7\%) were incomplete responders, and 4 (3.5\%) were treatment failures at the end of 1 year. Treatment failure cases who failed to improve with the standard regimen were treated as per the protocol mentioned in the methodology. Among the remaining 8 patients in whom immunosuppressants could not be started, 5 succumbed to death (2 presented with ALF and 3 with ACLF), 3 (who also presented with ACLF) were managed with supportive treatment and in them subsequently a steroid was started after stabilization. All these 8 patients were listed for liver transplantation but none received the transplant, because of a lack of resources and the prolonged waiting period at our setup.

Among the 27 elderly patients in whom immunosuppressants could be started, 14 (51.5\%) achieved complete remission, $11(40.7 \%)$ were incomplete responders, and 2 (7.5\%) were treatment failures (Table 3). Treatment failures in the elderly AIH group who failed to respond to the standard regimen were treated as per

Table 2. Histologic features in older and younger autoimmune hepatitis (AIH) patients

\begin{tabular}{|c|c|c|c|c|}
\hline & \multicolumn{2}{|c|}{ Age (years) } & \multirow[t]{2}{*}{ Total out of 146} & \multirow[t]{2}{*}{$P$-value } \\
\hline & $<60$ & $>60$ & & \\
\hline Interface hepatitis & $102 / 113(90.3 \%)$ & $27 / 33(81.8 \%)$ & $129(88.4 \%)$ & 0.183 \\
\hline Lymphoplasmacytic infiltrate & $104 / 113(92 \%)$ & $32 / 33(97 \%)$ & $136(93.2 \%)$ & 0.324 \\
\hline Rosette formation & $22 / 113(19.5 \%)$ & $8 / 33(24.2 \%)$ & $30(20.5 \%)$ & 0.55 \\
\hline Bile duct injury & $21 / 113(18.6 \%)$ & $7 / 33(21.2 \%)$ & $28(19.2 \%)$ & 0.736 \\
\hline Cholestasis & $48 / 113(42.5 \%)$ & $9 / 33(27.3 \%)$ & $57(39 \%)$ & 0.115 \\
\hline Cirrhosis & $69(56.6 \%)$ & $25(75.7 \%)$ & $94(73.7 \%)$ & $0.045 *$ \\
\hline
\end{tabular}

*Significant $p$ values. 
Table 3. Treatment response among older and young autoimmune hepatitis (AlH) patients in whom immunosuppressants could be started

\begin{tabular}{lcccc}
\hline & $\begin{array}{c}\text { Complete } \\
\text { response }\end{array}$ & $\begin{array}{c}\text { Incomplete } \\
\text { response }\end{array}$ & $\begin{array}{c}\text { Treatment } \\
\text { failure }\end{array}$ & $P$-value \\
\hline $\begin{array}{l}<60 \text { years } \\
(n=114)\end{array}$ & $75(65.6 \%)$ & $35(30.7 \%)$ & $4(3.7 \%)$ & 0.138 \\
\hline $\begin{array}{l}>60 \text { years } \\
(n=27)\end{array}$ & $14(51.5 \%)$ & $11(40.7 \%)$ & $2(7.5 \%)$ & 0.371 \\
\hline Total & $89(63.12 \%)$ & $46(32.62 \%)$ & $6(4.2 \%)$ & 0.165 \\
\hline
\end{tabular}

the protocol mentioned in the methodology. Among the remaining 6 elderly patients in whom immunosuppressants could not be started, 3 died (1 presented with ALF and 2 with ACLF) and the other 3 (who also presented with ACLF) were managed with supportive care and in them subsequently, a steroid was started after stabilization. All these 6 patients were listed for liver transplantation but none received the transplant.

Overall response to treatment at the end of 12 months was similar in both the groups $(p=0.165)$. Mortality was higher in the older group than the younger group, $3(9.1 \%)$ vs. 5 (4.09\%), although it was not statistically significant.

\section{Discussion}

In the present study, a wide spectrum of AIH was seen with varied presentation in young and older populations. In our study, $21.2 \%$ of AIH patients were diagnosed over the age of 60 , which is higher as compared to previously reported descriptive studies from India [11]. Being a tertiary care referral center, the diagnosis of AIH in the elderly was not delayed as compared to younger patients. This was in contrast with what was reported previously [13].

Chronic presentation was the commonest in both groups; however, ACLF like presentation was more frequent in the elderly population. This suggests that AIH runs a long subclinical course in the elderly before they are flared by some acute events resulting in ACLF. Acute events in most of our older population were acute viral hepatitis with hepatitis E virus being most common. Spontaneous AIH flare was also seen in a small number of cases. Though they presented like ACLF in our study, immunosuppressants could be started in the majority of them and their response to treatment was like those who presented like ACLF in the younger population. This is consistent with the reports of a large number of ACLF from India, where $\mathrm{AIH}$ accounts for $4.9 \%$ of all etiologies for underlying chronic disease and AIH as the etiology is similar in both survivors and non-survivors [14].
Symptom presentation greatly differed between younger and elder AIH patients in our study as compared to the previous studies $[13,15]$. Fever, arthralgia, fatigue and abdominal pain were much more common in younger patients. This could be a result of a stronger immunological reaction in younger patients as compared to the elderly in our study. Presence of other associated autoimmune diseases, especially autoimmune thyroiditis, in younger patients also points towards this fact. Though ascites was similar, spontaneous bacterial peritonitis was more common in the elderly. This could again be due to weakening immunity in elderly patients. However, other complications of cirrhosis were similar in both older and young patients.

The presence of autoimmune markers was similar in both the groups except for ASMA, which was more common in the elderly. When instituted at the appropriate time immunosuppression resulted in a treatment response (both complete and incomplete response) in $75.75 \%$ of the elderly and in $90.16 \%$ of younger patients, which were statistically similar. Mortality was similar in both groups. Thus, wherever possible immunosuppression should be started in all AIH patients irrespective of age. This was like previous studies of elderly AIH patients $[13,15]$. There have been reports in the past where treatment in the elderly has been withheld, although the response to treatment as shown by our study and others suggests that even older AIH patients are appropriate candidates for immunosuppression [16]. Although the treatment could not be started in around $18.2 \%$ of the elderly due to comorbidities, careful screening should be done as they benefit equally to younger patients from treatment. This may be attributed to a similar degree of liver injury on histology in both groups. Since in the present study the follow-up is only for 12 months, frequency of relapse and incidence of discontinuation of therapy due to side effects could not be determined among the two groups.

The limitations of our study included the lack of long-term follow-up beyond 12 months. Also, follow-up liver biopsy was not available in our older AIH subjects for comparison. The role of liver transplantation in those with treatment failure could not be determined in our center due to the resource-limited setup.

In conclusion, AIH in the elderly is not an uncommon diagnosis. Though the clinical presentation may differ from the younger population, their natural history and treatment outcomes are similar. Hence appropriate immunosuppression should be given early in them.

The study was approved by the institution's ethics committee. 


\section{Disclosure}

The authors report no conflict of interest.

\section{References}

1. Reuben A. A sheep in wolf's clothing. Hepatology 2003; 38: 1596-1601.

2. Lebovics E, Schaffner F, Klion FM, et al. Autoimmune chronic active hepatitis in postmenopausal women. Dig Dis Sci 1985; 30: 824-828.

3. Alvarez F, Berg PA, Bianchi FB, et al. International Autoimmune Hepatitis Group Report: review of criteria for diagnosis of autoimmune hepatitis. J Hepatol 1999; 31: 929-938.

4. Parker DR, Kingham JG. Type I autoimmune hepatitis is primarily a disease of later life. QJM 1997; 90: 289-296.

5. Donaldson PT, Czaja AJ. Genetic effects on susceptibility, clinical expression, and treatment outcome of type 1 autoimmune hepatitis. Clin Liver Dis 2002; 6: 419-437.

6. Donaldson PT, Doherty DG, Hayllar KM, et al. Susceptibility to autoimmune chronic active hepatitis: human leukocyte antigens DR4 and A1-B8-DR3 are independent risk factors. Hepatology 1991; 13: 701-706.

7. Verslype C, George C, Buchel E, et al. Diagnosis and treatment of autoimmune hepatitis at age 65 and older. Aliment Pharmacol Ther 2005; 21: 695-699.

8. Granito A, Muratori L, Pappas G, et al. Clinical features of type 1 autoimmune hepatitis in elderly Italian patients. Aliment Pharmacol Ther 2005; 21: 1273-1277.

9. Chen J, Eslick GD, Weltman M. Systematic review with meta-analysis: clinical manifestations and management of autoimmune hepatitis in the elderly. Aliment Pharmacol Ther 2014; 39: 117-124.

10. Sonthalia N, Rathi PM, Jain S, et al. Natural history and treatment outcomes of severe autoimmune hepatitis. J Clin Gastroenterol 2017; 51: 548-556.

11. Amarapurkar D, Dharod M, Amarapurkar A. Autoimmune hepatitis in India: single tertiary referral center experience. Trop Gastroenterol 2015; 36: 36-45.

12. Manns MP, Czaja AJ, Gorham JD, et al. Practice Guidelines of the American Association for the Study of Liver Diseases. Diagnosis and management of autoimmune hepatitis. Hepatology 2010; 51: 2193-2213.

13. Schramm C, Kanzler S, zum Büschenfelde KH, et al. Autoimmune hepatitis in the elderly. Am J Gastroenterol 2001; 96: 1587-1591.

14. Shalimar, Saraswat V, Singh P, et al. Acute-on-chronic liver failure in India: The Indian National Association for Study of the Liver consortium experience. J Gastroenterol Hepatol 2016; 31: 1742-1749.

15. Al-Chalabi T, Bocccato S, Portmann BC, et al. Autoimmune hepatitis (AIH) in the elderly: A systematic retrospective analysis of a large group of consecutive patients with definite AIH followed at a tertiary referral centre. J Hepatol 2006; 45: 575-583.

16. Newton JL, Burt AD, Park JB, et al. Autoimmune hepatitis in older patients. Age Ageing 1997; 26: 441-444. 